\title{
On the Average Power of Multiple Subcarrier Intensity Modulated Optical Signals: Nehari's Problem and Coding Bounds
}

\author{
Masoud Sharif and Babak Hassibi \\ Department of Electrical Engineering \\ California Institute of Technology \\ Pasadena, CA 91125 \\ Email: \{masoud,hassibi\}@ systems.caltech.edu
}

\begin{abstract}
Multiple subcarrier modulation (MSM) is an attractive technique for optical wireless communication for high speed applications. The main disadvantage of this scheme is its low average power efficiency which is an analogous problem to the high peak to mean envelope power ratio (PMEPR) of multicarrier signals. In this paper, we consider the achievable average power reduction of MSM signals by using optimized reserved carriers and coding methods. Based on Nehari's result we present a lower bound for the maximum average power of the signal after adding the reserved carriers. It is shown that the mean value of the average required power behaves very close to $\sqrt{2 n \log \log n}$ for a BPSK constellation where $n$ is the number of subcarriers. We then consider finding the optimum values for the carriers and the effect of having finite bandwidth for reserved carriers. In the next section, mainly based on recent coding results for the PMEPR of multicarrier signals, we show the existence of very high rate codes with average power of $O(\sqrt{n \log n})$ for large values of $n$, and furthermore the existence of codes with non-vanishing to zero rate and average power of $O(\sqrt{n})$ asymptotically.
\end{abstract}

\section{INTRODUCTION}

Intensity modulation (IM) with direct detection (DD) is an attractive technique for high speed wireless optical communications [1]. Multiple subcarrier modulation (MSM) has been proposed in IM/DD systems due to its immunity to intersymbol interference in multipath channels. In this scheme, the multiple subcarrier signal is modulated onto the optical carrier by intensity modulation.

The main disadvantage of MSM with IM/DD is its low optical average power efficiency which is essentially the same problem as the high peak to mean envelope power ratio (PMEPR) of multicarrier signals in RF communications: high amplitude variations. Since the optical intensity or instantaneous power should be always positive, the signal should have a dc component to guarantee that the minimum of the signal is greater than zero. Since this dc component is proportional to the average optical power, as the number of carriers $n$,

This work was supported in part by the National Science Foundation under grant no. CCR-0133818, by the office of Naval Research under grant no. N00014-02-1-0578, and by Caltech's Lee Center for Advanced Networking. increases and the signal exhibits smaller minimum values, the required average power of the system will be increased.

Previously, in [2] and [3], the clipping method has been considered to increase the average power efficiency. Recently, You and Kahn [4] proposed block coding and tone reservation to maximize the minimum value of the multiple subcarrier signal. In the coding method, coding is used to decrease the dc component that guarantees the positivity of the signal. On the other hand, tone reservation, is a method in which we use dummy carriers with optimized amplitude just to increase the average power efficiency of the system. It is worth noting that the tone reservation problem here is a bit different from that of OFDM (Orthogonal Frequency Division Multiplexing) in the sense that we are just interested in the maximization of the minimum value of the signal unlike OFDM in which we are interested in the ratio of the maximum of the signal to the average power [5].

In this paper, we address the achievable average power reduction by using optimum reserved tones appended at the end of the signal and also using coding methods. We initially relate Nehari's result on the estimation of a causal function by an anti-causal function to the average power reduction of the multicarrier signals, and we propose a bound on the maximum reduction of the dc component. We then discuss how to optimize the dummy tones in order to reduce the average power.

In the next part of the paper, we use some recent coding results for the PMEPR of OFDM signals, to present asymptotic bounds on the average power reduction of the optical MSM signals [6], [7]. We first show that in an uncoded system, restricting the transmit codewords to codewords with average power requirement of $O(\sqrt{n \log n})$ does not reduce the number of codeowords substantially for large number of subcarriers where $\log (\cdot)$ is the natural logarithm. Furthermore, we show the existence of codes with average required power of $O(\sqrt{n})$ and nonzero rate, asymptotically [7].

In summary, for large values of $n$, Theorem 2 implies that with probability one the average power required is $O(\sqrt{n \log n})$ for a large class of QAM/PSK constellations. 
Based on Nehari's Theorem, Corollary 1 states that by using reserved carriers appended at the end of signal's spectrum, the mean value of the required average power can be reduced to at most $\sqrt{2 n \log \log n}$ for BPSK constellations. Finally we state the existence of codes with average power requirement of $O(\sqrt{n})$ in Theorem 3.

The paper is organized as follows. In Section II, we describe the formulation of the problem and multiple subcarrier modulations. We then briefly discuss methods to reduce the average power and the open problems raised in [4]. Section III presents Nehari's theorem and its implication on the average power reduction using reserved carries at the end of the signal's spectrum. Section IV considers the coding method and its achievable reduction on the average power. Finally, Section $\mathrm{V}$ concludes the paper with some open problems.

\section{Multiple Subcarrier Modulation}

Similar to the OFDM signals, MSM signals consist of $n$ subcarriers where the $i$ 'th subcarrier is modulated by $c_{i}$ which is chosen from a constellation like BPSK or more generally QAM/PSK. Therefore, the MSM signal can be written as

$$
s(\tau)=\operatorname{Re}\left\{\sum_{i=1}^{n} c_{i} e^{j \omega_{i} \tau}\right\} g(\tau) \quad 0 \leq \tau<T,
$$

where $g(t)$ is the transmit pulse shape, $T$ is the symbol duration, and $\omega_{i}$ 's are carrier frequencies. Throughout this paper as in [4], we will consider a rectangular pulse shape, i.e. $g(\tau)=u(\tau)-u(\tau-T)$, and dense packing for the subcarriers which implies that,

$$
\omega_{i}=i \frac{2 \pi}{T}, \quad i=1, \ldots, n .
$$

Without loss of generality, to simplify the mathematical formulation, we normalize the time axis by $T$ to get,

$$
s(t)=\operatorname{Re}\left\{\sum_{i=1}^{n} c_{i} e^{j 2 \pi i t}\right\} \quad 0 \leq t \leq 1 .
$$

To make the transmitted signal positive, we have to add a signal $b(t)$ and guarantee that $b(t)+s(t)$ is non-negative. Generally, for each codeword $\left(c_{1}, \ldots, c_{n}\right), b(t)$ consists of a dc part $b_{0}$, and a time variant part $b_{1}(t)$. Clearly since the transmitted signal after amplifications is $A(s(t)+b(t))$, the average optical power will be,

$$
P=A \int_{0}^{2 \pi}(s(t)+b(t)) d t=A 2 \pi b_{0},
$$

since the average of $s(t)$ and $b_{1}(t)$ are zero. In order to analyse the minimum average power required, the minimum value of $s(t)+b_{1}(t)$ should be maximized. In this paper, we will consider a stronger condition and we minimize the maximum value of $\left|\sum_{i=1}^{n} c_{i} e^{j 2 \pi t}+\bar{b}(t)\right|$ where $b_{1}(t)=\operatorname{Re}\{\bar{b}(t)\}$. More specifically, if we define

$$
T(z)=\sum_{i=1}^{n} c_{i} z^{i}
$$

to guarantee the positiveness of the transmitted signal, we may consider the minimization of the maximum of $\left|T\left(e^{j 2 \pi t}\right)+\bar{b}(t)\right|$ which limits the variations of the signal both in the negative and positive sides. Therefore, the average power requirement is proportional to the maximum of $\left|T\left(e^{j 2 \pi t}\right)+\bar{b}(t)\right|$. From a practical point of view, this restriction on the envelope rather than the real part of the signal will both reduce the average power requirement and eliminate high instantaneous intensity peaks from the transmitted signal.

A well-known method in OFDM systems is to use optimized reserved carriers to minimize the ratio of the peak to average power of the multicarrier signal [5]. However, here for MSM optical signals, we just need to maximize the minimum of the signal regardless of the amplitude of the dummy tones. Therefore, since the average power does not play any role, we can use Nehari's theorem to investigate the maximum average power reduction when we are allowed to use reserved carriers with frequencies higher than $n$ [8]. The achievable average power reduction with reserved carriers is addressed in Section III.

In [4], block coding is also proposed to reduce the average transmitted power. The idea in this method is basically to map $k$ information symbols $\left(\zeta_{1}, \ldots, \zeta_{k}\right)$ to the transmitted codeword $C=\left(c_{1}, \ldots, c_{n}\right)$ where $n>k$, and all transmitted codewords are chosen such that the minimum value of $s(t)$ is greater than some desired value. In Section IV, we propose bounds on the achievable average power reduction by coding mainly based on recent results on the PMEPR of OFDM signals [6], [7], [9].

\section{Nehari's Problem and Its ImPlications ON THE RESERVED CARRIER METHOD}

As explained in the previous section, one method to increase the average power efficiency of MSM signals is to add dummy carriers with optimum values at the end of the signal. Therefore we can formulate this problem as the following:

Problem Statement: Let $c_{i}$ 's be given, $T(z)$ as in (5) and $B(z)=\sum_{i=n+1}^{M} b_{i} z^{i}$, find the optimum values of $b_{i}$ 's and the best $\gamma$ such that for all $c_{i}$ 's, we have

$$
\|T(z)-B(z)\|_{\infty} \leq \gamma
$$

A slightly different problem in functional analysis, known as Nehari's problem, states the following result:

Theorem 1: (Nehari's Theorem [8]) Let $T_{1}(z)=$ $\sum_{i=1}^{n} c_{n-i+1} z^{-i}$ be an anticausal function. Then,

$$
\inf _{\operatorname{causal} K_{1}(z)}\left\|T_{1}(z)-K_{1}(z)\right\|_{\infty}=\sigma\left(\mathcal{H}_{T}\right)
$$

where $K_{1}(z)=\sum_{i=0}^{\infty} d_{i} z^{i}$ and $\mathcal{H}_{T}$ is the Hankel operator,

$$
\mathcal{H}_{T}=\left[\begin{array}{cccc}
c_{n} & c_{n-1} & \ldots & c_{1} \\
c_{n-1} & \ldots & c_{1} & 0 \\
\vdots & & & \\
c_{1} & 0 & \ldots &
\end{array}\right]
$$

and $\sigma(\cdot)$ denotes the maximum singular value of its argument. 
Remark 1: It is worth mentioning that Nehari also showed that the optimum values of the $d_{i}$ 's satisfy the following:

$$
\gamma z \prod_{i=1}^{n-1} \frac{\beta_{i}+z}{1+\beta_{i}^{*} z}=\sum_{i=1}^{n} c_{n-i} z^{i}+\sum_{i=0}^{\infty} d_{i} z^{n+i+1}
$$

where $\left|\beta_{i}\right|<1$ for all $i$ 's. Therefore, the sequence $d_{i}$ is an exponentially decreasing function of $i$, and practically, we will be able to truncate the infinite series $K_{1}(z)$ to its first $M-n$ coefficients.

Remark 2: To find the solution to Nehrai's problem, we can use Theorem 12.8 .2 of [10] to find $K_{1}(z)$ numerically. We describe the detail of this computation in the appendix.

Comparing the statement of the problem and Nehari's theorem, we can easily state the following corollary to find a lower bound for maximum average power reduction by using reserved carriers at the end of the signal.

Corollary 1: Let $T(z)$ be as in (5) and $B(z)=$ $\sum_{i=n+1}^{M} b_{i} z^{i}$. Then for all $z$ over the unit circle and for any $B(z)$, we have

$$
\max _{\left(c_{1}, \ldots, c_{n}\right)} \sigma\left(\mathcal{H}_{T}\right) \leq\|T(z)-B(z)\|_{\infty} .
$$

The bound can be achieved by choosing $B(z)$ with infinite length according to Nehari's Theorem.

Proof: This is a straightforward consequence of Nehari's result by simply considering $T(z)=z^{n+1} T_{1}(z)$ where $T(z)$ is as defined in (5). For all $z$ on the unit circle, Theorem 1 implies that,

$$
\begin{aligned}
\sigma\left(\mathcal{H}_{T}\right) & =\left\|z^{-n-1} T(z)-\sum_{i=0}^{\infty} d_{i} z^{i}\right\|_{\infty} \\
& =\left\|T(z)-\sum_{i=n+1}^{\infty} d_{i-n-1} z^{i}\right\|_{\infty} \\
& \leq\left\|T(z)-\sum_{i=n+1}^{M} b_{i} z^{i}\right\|_{\infty}
\end{aligned}
$$

where the inequality follows from the fact that the optimum sequence $d_{i}$ can obviously have a smaller norm than the special case where coefficients of $z^{M+1}$ and higher are zero. The result follows immediately by maximizing both sides of (11) over all the codewords $\left(c_{1}, \ldots, c_{n}\right)$.

For simplicity, from now on in this section, we consider $c_{i}$ 's chosen from a BPSK constellation. The numerical results here are easily extendible to other constellations as well. To find a better insight to the result of Corollary 1, we can easily bound the maximum singular value of $\mathcal{H}_{T}$ by using the fact that the sum square of the eigenvalues of a matrix is equal to the Frobenius norm of the matrix. This implies that

$$
\max \sigma\left(\mathcal{H}_{T}\right) \leq\left\{\sum_{i=1}^{n} i\right\}^{1 / 2}=\sqrt{\frac{n(n+1)}{2}} .
$$

A lower bound on $\sigma\left(\mathcal{H}_{T}\right)$ can be obtained by considering the codeword $C^{\prime}=(1,1, \ldots, 1)$. The lower and upper bounds are shown in Fig. 1. Numerically it can be verified that for

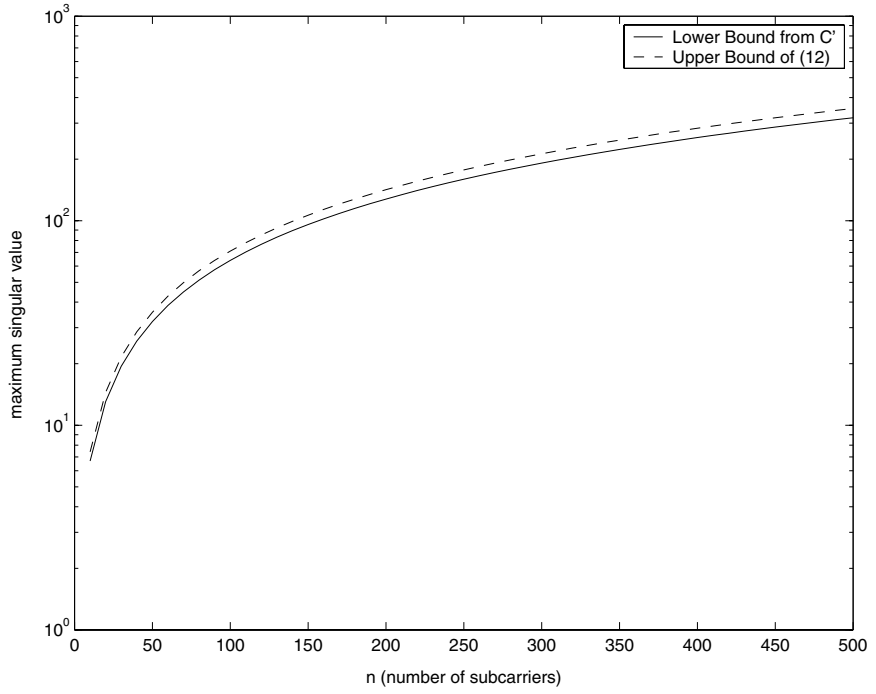

Fig. 1. Lower and Upper bounds on the maximum singular value of the random BPSK Hankel matrix.

$n \leq 24$ the maximum of $\sigma\left(\mathcal{H}_{T}\right)$ is indeed achieved by $C^{\prime}$.

Therefore the worst case improvement is not that much and it is just from $n$ to $\sqrt{n(n+1) / 2}$. However we can also evaluate the average of the maximum singular value of $\mathcal{H}_{T}$ over all possible codewords. Fig. 2 shows the average of $\max \sigma\left(\mathcal{H}_{T}\right)$ for BPSK and when the number of subcarriers varies from 10 to 500 . It can be observed numerically that the average behaves very close to $\sqrt{2 n \log \log n}$ that is much better than $\sqrt{n \log n}$ which is the average of $\|T(z)\|_{\infty}$ before using dummy tones asymptotically [6](see also Section IV).

To further investigate the improvement by using reserved carriers, Fig. 3 shows the complementary cumulative distribution function of the maximum, $\operatorname{Pr}\left\{\left\|T(z)-K_{1}(z)\right\|_{\infty}>\lambda\right\}$ before and after using optimum dummy tones for $n=128$. We also consider the effect of truncating $K_{1}(z)$ to a finite degree polynomial and its effect on the distribution function. It is clear that we have quite a lot of improvement with 20 reserved carriers and further increasing the number of tones does not improve the distribution as much as before.

It is worth mentioning that the problem of finding the optimum values for a finite number of reserved carriers can be exactly solved by invoking the Bounded Real Lemma and using the LMI (Linear Matrix Inequality) optimization [11], [10](Theorem 12.6.6). However, it can be shown that the complexity of the LMI is $O\left((n+L)^{6}\right)$ where $L$ is the number of reserved tones. On the other hand, all that the computation of $K_{1}(z)$ requires is a matrix inversion and a maximum singular value computation which has $O\left(n^{3}\right)$ complexity. Therefore truncating the Nehari solution to finite length $L$ seems to be a more practical way of finding good values for reserved carriers. 


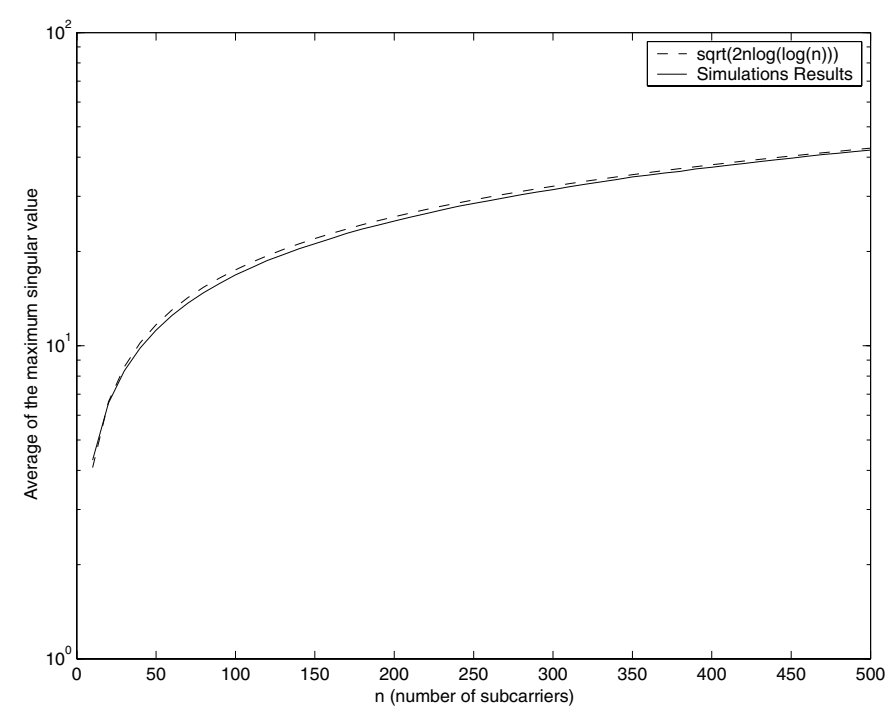

Fig. 2. Average of the maximum singular value of the random Hankel matrix and $\sqrt{2 n \log \log n}$.

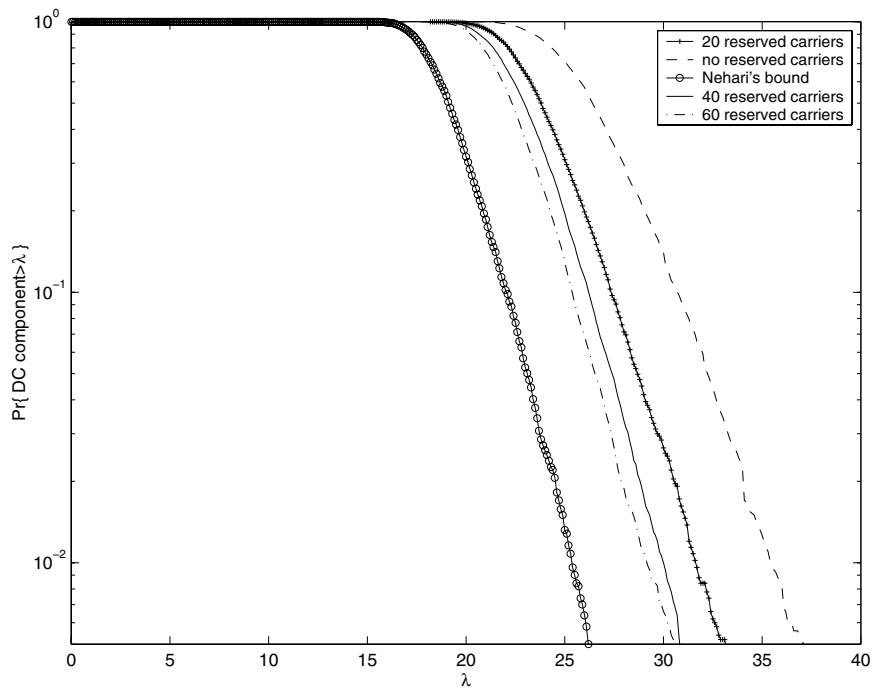

Fig. 3. Distribution function of the required dc component with and without using reserved carriers and the effect of truncation of $K_{1}(z)$.

\section{Average Power Reduction Using Coding}

Another promising technique to reduce $\|T(z)\|_{\infty}$ is to use coding. To clarify the idea, let's consider the following example. Let the $c_{i}$ 's be chosen from QPSK. Then for an uncoded system, we have a set $\mathcal{C}$ with $2^{2 n}$ possible codewords $\left(c_{1}, \ldots, c_{n}\right)$, and the average power required to make all the signals positive is greater than $n$. Now instead of sending all of the codewords, we consider a subset $\mathcal{C}^{\prime}$ of $\mathcal{C}$ in which all the codewords have $\|T(z)\|_{\infty} \leq f(n)$ where $f(n)$ is an arbitrary function less than $n$, say $\log n$ or $\sqrt{n}$. Therefore, by adding a dc component equal to $\|T(z)\|_{\infty}$, we can reduce the average power from $n$ to $f(n)$ at the price of reducing the rate from
2 to $\frac{1}{n} \log _{2}\left|\mathcal{C}^{\prime}\right|$ where the rate of a code $\mathcal{C}$ is defined as,

$$
R=\frac{1}{n} \log _{2}|\mathcal{C}|
$$

and $|\mathcal{C}|$ is the cardinality of the set $\mathcal{C}$.

The goal of this section is to quantify the trade-off between rate and average power reduction of the code for sufficiently large values of $n$. The results are simple consequences of analogous results for the PMEPR of OFDM.

Theorem 2: Let $T(z)$ be as in (5) and $C=\left(c_{1}, \ldots, c_{n}\right)$ be a random codeword where $c_{i}=a_{i}+j b_{i}$ is chosen independently and identically from the following constellations: ( $i$ ) QAM where $a_{i}$ 's and $b_{i}$ 's are independent with even distributions, (ii) PSK in which the distribution of $c_{i}$ is invariant under $\pi / 2$ rotation. Then, with probability one

$$
\lim _{n \rightarrow \infty} \frac{\|T(z)\|_{\infty}}{\sqrt{n E_{a v} \log n}}=1
$$

where $E_{a v}=E\left\{\left|c_{i}\right|^{2}\right\}$.

Proof: The proof is based on the asymptotic analysis of the distribution of the maximum of the random multicarrier signals. Refer to [6] and [12] for a complete proof.

Theorem 2 implies that almost all the codewords in an uncoded system have $\|T(z)\|_{\infty} \leq \sqrt{n E_{a v} \log n}$, and restricting the transmit codewords to the codewords with $\|T(z)\|_{\infty} \leq$ $\sqrt{n E_{a v} \log n}$ does not reduce the rate for large values of $n$ [6]. Now the question would be what will be happen to the rate of the code if we want to further reduce the average power from $O(\sqrt{n \log n})$ to say $O(\sqrt{n})$ ?

An analogous question for PMEPR reduction has been answered recently by choosing an optimum sign for each subcarrier in order to reduce $\max \left|\sum_{i=1}^{n} c_{i} e^{j 2 \pi i t}\right|$ [7], [9]. Given the vector $\left(c_{1}, \ldots, c_{n}\right)$, it is in fact shown that by properly choosing $\epsilon_{i} \in\{-1,+1\}$, we can reduce $\max \left|\sum_{i=1}^{n} \epsilon_{i} c_{i} e^{j 2 \pi i t}\right|$ and achieve the dc component of $O(\sqrt{n})$. In the following theorem, we state the existence of exponentially many codewords with average power of $O(\sqrt{n})$ for large values of $n$.

Theorem 3: Let the $c_{i}$ 's be chosen from a symmetric $q$ ary constellation in which if $A$ is in the constellation then $-A$ has to be in the constellation. Then for large values of $n$, there exists a code $\mathcal{C}^{\prime}$, with rate greater than or equal to $\log _{2} q-1$ and

$$
\max _{C \in \mathcal{C}^{\prime}}\|T(z)\|_{\infty}<\alpha \sqrt{n}
$$

where $C=\left(c_{1}, \ldots, c_{n}\right)$ is a codeword in the code set $\mathcal{C}^{\prime}$, and $\alpha$ is a constant independent of $n$.

Proof: The proof is based on choosing the optimum sign vector $\left(\epsilon_{1}, \ldots, \epsilon_{n}\right)$ for any codeword $C$ to minimize $\|T(z)\|_{\infty}$ and achieve $O(\sqrt{n})$. Refer to [7] for the proof.

So for large values of $n$, there exist exponentially many codewords with average power of $O(\sqrt{n})$. In other words, the rate hit to reduce the average power from $O(n)$ to $O(\sqrt{n})$ is at most $1 \mathrm{bit} / \mathrm{sec}$. Meanwhile, Theorem 2 implies that the ratio of the number of these codewords to the total number of admissible codewords is zero.

It is also worth mentioning that in [9], an algorithm is 
proposed to design the signs and reduce $\|T(z)\|_{\infty}$ from $O(n)$ to $O(\sqrt{n \log n})$. The algorithm in fact suggests a deterministic construction of a code with rate $\log _{2} q-1$ to reduce the average power from $O(n)$ to $O(\sqrt{n \log n})$.

\section{Conclusions}

In this paper we consider the achievable average power reduction for multiple subcarrier IM/DD optical signals by using dummy tones with optimum value at the end of the signal and also using coding method. Based on Nehari's theorem, we present a bound on the maximum average power reduction using optimum dummy tones. In the second section, we used recent coding results for the PMEPR of OFDM signals to introduce coding bounds for average power reduction. We showed the existence of codes with average power of $O(\sqrt{n \log n})$ that can be constructed with probabilistic methods. We further presented the existence of codes with high rate (not vanishing to zero) and average power of $O(\sqrt{n})$.

Several problems remain to be answered. We considered the problem of reserved carriers when they are placed at frequencies larger than $n$, it would be interesting to investigate the effect of changing their place on the average power reduction. As the numerical results in Fig. 2 suggest, it is intriguing to show that the average of the maximum singular value of random BPSK Hankel matrices is equal to $\sqrt{2 n \log \log n}$. In fact similar to Eq. (14), we suspect that for large values of $n$ with probability 1 , the maximum singular value is equal to $\sqrt{2 n \log \log n}$.

\section{APPENDIX}

In this appendix, we outline the derivations of the solution to the Nehari's problem, $K_{1}(z)$. The procedure is based on Section 12.8 of [10].

Let $T(z)$ and $\mathcal{H}_{T}$ be defined as in (5) and (8), then define $H=\left[c_{n}, \ldots, c_{1}\right], G^{t}=[1,0, \ldots, 0]$,

$$
F=\left[\begin{array}{cccc}
0 & \ldots & 0 & \\
1 & 0 & \ldots & \\
0 & 1 & \ldots & \\
\vdots & \vdots & & \\
0 & \ldots & 1 & 0
\end{array}\right]
$$

and $P=\mathcal{H}_{T}^{2}\left(\gamma^{2}-\mathcal{H}_{T}^{2}\right)^{-1}$, where $\gamma$ is a real number greater than $\sigma\left(\mathcal{H}_{T}\right)$. Then the matrices $R_{e}$ and $K_{p}$ can be defined as,

$$
\begin{gathered}
R_{e}=\left[\begin{array}{cc}
1 & 0 \\
0 & -\gamma^{2}+H H^{*}
\end{array}\right]+\left[\begin{array}{c}
-G^{*} \\
H F^{*}
\end{array}\right] P\left[\begin{array}{ll}
-G & F H^{*}
\end{array}\right] \\
K_{p}=\left[\begin{array}{ll}
-F^{*} P G & F^{*} P F H^{*}+H^{*}
\end{array}\right] R_{e}^{-1}
\end{gathered}
$$

Then $K_{1}(z)=-L_{21}\left(z^{-1}\right) L_{11}^{-1}\left(z^{-1}\right)$ where,

$$
L_{11}^{-1}(z)=a_{0}\left[I-a_{0}^{2} G^{*}\left(z I-F^{*}+a_{0}^{2} F^{*} P G G^{*}\right)^{-1} F^{*} P G\right]
$$

$L_{21}(z)=a_{0}^{-1}\left\{\left[\begin{array}{ll}0 & 1\end{array}\right]+H F^{*}\left(z I-F^{*}\right)^{-1} K_{p}\right\}\left[\begin{array}{c}1 \\ -H F^{*} P G\end{array}\right]$

where $a_{0}=\left(1+G^{*} P G\right)^{-\frac{1}{2}}$. By further using the identity $\left(I-z^{-1} A\right)^{-1}=\sum_{i=0}^{\infty} z^{-i} A^{i}$ for any stable matrix $A$, we get,

$$
\begin{aligned}
L_{11}^{-1}\left(z^{-1}\right) & =\sum_{i=0}^{\infty} \alpha_{i} z^{i} \\
L_{21}\left(z^{-1}\right) & =\sum_{i=0}^{n} \beta_{i} z^{i}
\end{aligned}
$$

where $\alpha_{i}$ and $\beta_{i}$ 's can be numerically evaluated and $K_{1}(z)$ will be derived.

\section{REFERENCES}

[1] J. R. Barry, Wireless Infrared Communications, Boston, MA: kluwer, 1994.

[2] Q. Pan and R. J. Green, "Preclipping AM/QAM hybrid lightwave systems with bandstop filtering," IEEE Photon. Tech. Let., vol. 8, no. 8, pp. 1079-1081, Aug. 1996.

[3] Q. Shin, "Error performance of OFDM-QAM in subcarrier multiplexed fiber-optic transmission," IEEE Photon. Tech. Let., vol. 9, no. 6, pp. 845-847, June 1997.

[4] R. You and J. M. Kahn, "Average power reduction techniques for multiple subcarrier inrensity modulated optical signals," IEEE Trans. Comm., vol. 49, no. 12, pp. 2164-2171, Dec. 2001.

[5] J. Tellado and J. M. Cioffi, "Efficient algorithms for reducing PAR in multicarrier systems," in Proc. IEEE Inter. Symp. Info., August 1998, p. 191.

[6] M. Sharif and B. Hassibi, "On multicarrier signals where the PMEPR of a random codeword is asymptotically $\log n$," submitted to IEEE Trans. Inform., 2002.

[7] M. Sharif and B. Hassibi, "On the existence of codes with constant peak to mean envelope power ratio," submitted to IEEE Int. Symp. Info. Theo., 2002.

[8] Z. Nehari, "On bounded bilinear forms," The Annals of Mathematics, vol. 65, no. 1, pp. 153-162, 1957.

[9] M. Sharif and B. Hassibi, "A deterministic algorithm that achieves PMEPR of $c \log n$ for multicarrier signals," to be presented in IEEE ICASSP 2003.

[10] B. Hassibi, A. H. Sayed, and T. Kailath, Indefinite-Quadratic estimation and control: a unified approach to $\mathrm{H}^{2}$ and $\mathrm{H}^{\infty}$ theories, SIAM studies in applied and numerical mathematics, 1999.

[11] Shao po Wu, S. Boyd, and L. Vandenberghe, "FIR filter design via semidefinite programming and spectral factorization," in Proc. 35th IEEE Conf. on Decision and Contol, 1996, pp. 271-276.

[12] G. Halasz, "On the result of Salem and Zygmund concerning random polynomials," Studia Scien. Math. Hung., pp. 369-377, 1973. 